Notions de registre et de cadre de rationalité en inter-didactique des mathématiques et de la physique

Didier Malafosse, Alain Lerouge et Jean-Michel Dusseau

\title{
CpenEdition
}

Journals

Édition électronique

URL : http://journals.openedition.org/trema/1629

DOI : $10.4000 /$ trema.1629

ISSN : 2107-0997

Éditeur

Faculté d'Éducation de l'université de Montpellier

Édition imprimée

Date de publication : 1 décembre 2000

Pagination : 49-60

ISSN : 1167-315X

Référence électronique

Didier Malafosse, Alain Lerouge et Jean-Michel Dusseau, « Notions de registre et de cadre de rationalité en inter-didactique des mathématiques et de la physique », Tréma [En ligne], 18 | 2000, mis en ligne le 01 décembre 2000, consulté le 12 juin 2020. URL : http://journals.openedition.org/trema/ 1629 ; DOI : https://doi.org/10.4000/trema.1629

Ce document a été généré automatiquement le 12 juin 2020

Trema 


\section{Notions de registre et de cadre de rationalité en inter-didactique des mathématiques et de la physique}

Didier Malafosse, Alain Lerouge et Jean-Michel Dusseau

1 Les professeurs qui enseignent les sciences physiques et leurs collègues de mathématiques sont souvent en désaccord lorsqu'il s'agit d'analyser les difficultés rencontrées par leurs élèves dans le transfert de connaissances d'une discipline à l'autre. Ce constat nous a amenés à développer un programme de recherche en interdidactique des mathématiques et de la physique (Malafosse, Lerouge \& Dusseau, 2000, 2001) visant à travailler, d'un point de vue croisé, les processus de conceptualisation mis en œuvre dans les deux disciplines.

2 Si au niveau historique, la seule évocation des noms de Descartes, de Pascal, ou de Fourrier, montre à quel point les développements respectifs des mathématiques et de la physique sont intimement liés, au niveau didactique, les rapprochements entre les deux disciplines sont balbutiants, et rares sont les travaux de recherche qui abordent la problématique de la conceptualisation scientifique d'un point de vue interdisciplinaire. Dans le courant émergeant du rapprochement des didactiques, plusieurs stratégies se font jour. Une tendance consiste à choisir une notion didactique pertinente dans une discipline et à la mettre à l'épreuve dans l'autre dans une perspective de transfert. La notion didactique considérée est alors l'objet de l'étude inter-didactique (Fig. 1). 




Figure 1

Stratégie de la notion-objet.

Une autre tendance consiste à analyser les processus de conceptualisation des élèves sur des concepts transversaux, et à mener une étude différentielle de ces processus dans chaque discipline. Dans ce cas, les notions didactiques auxquelles on a recours sont mises en œuvre en tant qu'outils d'analyse des phénomènes observés (Fig. 2).

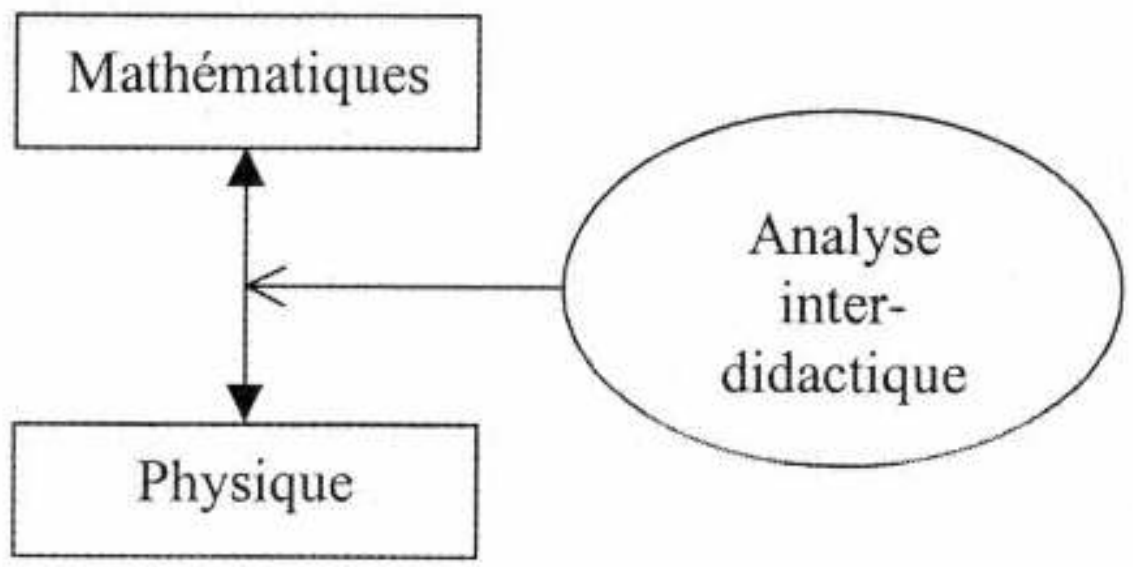

Figure 2

Stratégie de la notion-outil.

Dans le contexte d'une étude sur la proportionnalité dans l'enseignement de la loi d'Ohm en classe de Troisième, notre démarche de recherche a consisté à utiliser de manière dialectique ces deux stratégies. Dans un premier temps, la comparaison des processus cognitifs développés en mathématiques et en physique nous a amenés à mobiliser un certain nombre de notions didactiques comme outils aptes à rendre compte des obstacles induits par le passage d'une discipline à l'autre. Dans un second temps, nous avons pris ces notions comme objet d'étude dans la perspective de les faire évoluer pour les intégrer entre elles dans un modèle inter-didactique. Au terme de plusieurs cycles expérimentaux outil - objet, nous avons stabilisé notre approche sur deux notions issues de la didactique des mathématiques : celle de registre sémiotique (Duval, 1995), et de cadre de rationalité (Lerouge, 1992).

L'objet de cet article est de présenter ces notions dans leur contexte d'origine, puis d'en montrer l'intégration dans l'analyse de processus de conceptualisation en interdidactique des mathématiques et de la physique. 


\section{Les registres sémiotiques}

5 La notion de registre sémiotique a été introduite en didactique des mathématiques par R. Duval, qui n'a pas envisagé son transfert à d'autres contextes didactiques.

\section{Représentations sémiotiques et représentations mentales}

R. Duval (1993) insiste sur le fait que les représentations sémiotiques sont plus qu'un «simple moyen d'extériorisation des représentations mentales à des fins de communication »et qu'elles interviennent à plusieurs niveaux de l'activité cognitive : le développement des représentations mentales, l'accomplissement des différentes fonctions cognitives, et la production de connaissances nouvelles.

"On ne peut pas faire comme si les représentations sémiotiques étaient simplement subordonnées aux représentations mentales, puisque le développement des secondes dépend de l'intériorisation des premières et que seules les représentations sémiotiques permettent de remplir certaines fonctions cognitives essentielles » (Duval, ibid).

\section{Registre sémiotique}

7 Après avoir postulé que "les représentations sémiotiques sont des représentations dont la production ne peut pas se faire sans la mobilisation d'un système sémiotique »(1995a), R. Duval caractérise un registre sémiotique comme un système sémiotique "permettant de remplir[à la fois] les trois fonctions cognitives fondamentales:»la «fonction de communication", la «fonction de traitement "[c'est-à-dire la transformation de la représentation sans changer de système sémiotique], et la "fonction d'objectivation" [permettant à la fois la prise de conscience de l'existence de la représentation en tant que telle, et sa conversion dans un autre système sémiotique].

8 Les activités mathématiques nécessitent que l'élève fasse appel à plusieurs registres sémiotiques, par exemple le registre de la langue naturelle, celui de l'écriture algébrique, ou celui des représentations graphiques cartésiennes. L'apprentissage se fonde pour une part sur le jeu entre ces différents registres, qu'il apparaît indispensable de coordonner.

\section{Coordination des registres sémiotiques}

9 Les travaux de R. Duval ont mis à mal l'idée généralement admise que le choix pertinent d'un seul registre sémiotique permettait la compréhension du contenu conceptuel représenté.

"La compréhension (intégrative) d'un contenu conceptuel repose sur la coordination d'au moins deux registres de représentation, et cette coordination se manifeste par la rapidité et la spontanéité de l'activité de conversion». Mais "cette coordination est loin d'être naturelle. Et elle ne semble pas pouvoir se réaliser dans le cadre d'un enseignement principalement déterminé par des contenus conceptuels » (Duval, 1995a).

10 Cet auteur met en évidence à quel point le cloisonnement des registres chez les élèves les conduit à ne pas reconnaître "le même objet à travers des représentations qui en sont données dans des systèmes sémiotiques différents ».Pour lever ce cloisonnement il a recours à la notion de «congruence » entre deux représentations sémiotiquement différentes 
et qui représentent au moins partiellement le même contenu. Deux représentations sont «congruentes" pour l'apprenant s'il est capable d'établir une correspondance sémantique biunivoque entre les éléments signifiants de ces représentations dans leurs registres sémiotiques respectifs. Lorsque des représentations ne sont pas congruentes, par manque "d'apprentissage préalable concernant les spécificités sémiotiques de formation et de traitement de représentation qui sont propres à chacun des registres en présence ",non seulement le temps de traitement est important, mais encore la conversion se révèle " impossible à effectuer, ou même à comprendre ".

\section{Complémentarité des registres et économie de traitement}

11 A cette idée de conversion de registre nécessaire à la compréhension conceptuelle s'ajoute celle de complémentarité des approches qu'apporte l'utilisation de registres sémiotiques différents. Bresson (1987) a montré que, dès que l'on choisit un registre sémiotique pour représenter un contenu cognitif, les possibilités et les contraintes sémiotiques du registre conduisent à ne sélectionner que certains éléments significatifs du contenu. Il s'ensuit que la représentation sémiotique ne représente qu'une partie de ce contenu, et que ce n'est que par le rapprochement des représentations d'un même contenu dans divers registres que l'on peut espérer le représenter dans toute sa complexité cognitive.

Enfin, la maîtrise de plusieurs registres permet d'en changer de façon à réaliser les traitements cognitifs de la manière la plus économique possible. Pour s'en convaincre, il suffit d'essayer de formuler dans le registre de la langue naturelle un raisonnement de type calcul. On constatera immédiatement l'économie réalisée en se plaçant dans le registre de l'écriture algébrique.

\section{Les cadres de rationalité}

En référence aux travaux de R. Douady (1984), A. Lerouge (1992), définit un « un cadre de rationalité "comme "un ensemble cohérent de fonctionnement de la pensée caractérisé par quatre composantes: son monde d'objets, ses champs de concepts, son mode de rationalité, et ses registres de signifiants ».

Depuis une dizaine d'année, cette définition a sensiblement évolué, et nous retenons aujourd'hui, les quatre composantes suivantes :

-1. les objets (nous reviendrons sur ce terme) du cadre ;

-2. le type de processus de conceptualisation (familier ou scientifique au sens de L.S.

Vygotski) mis en œuvre ;

-3. les règles de rationalité (ce sont les règles de validation, mais aussi les règles de raisonnement) ;

-4. et enfin les registres sémiotiques support de conceptualisation et de communication.

Par exemple, analysant les processus de conceptualisation de la droite au collège, A. Lerouge (2000) en arrive à la conclusion qu'il existe entre le cadre familier d'un élève de collège et le cadre culturel de la géométrie enseignée quatre niveaux de ruptures :

-1. « une rupture d'objet entre la droite matérielle et la droite idéelle ;

-2. une rupture de processus de conceptualisation entre la conceptualisation spontanée et la conceptualisation scientifique ; 
-3. une rupture de processus de validation entre le constat figurai et la démonstration ;

-4. et une rupture de registre sémiotique entre le dessin et la figure ».

\section{Extension de la notion}

Initialement limitée à l'analyse des ruptures entre le versant sujet et le versant culturel dans l'apprentissage des mathématiques, nous avons étendu la notion de cadre de rationalité à la comparaison de deux cadres culturels, notamment celui des mathématiques et celui de la physique. Cela nous a amenés à travailler le champ conceptuel de la proportionnalité au travers de trois cadres de rationalité : les cadres culturels des mathématiques et des sciences physiques, et le cadre personnel de l'apprenant au regard de la parcellisation de l'espace de pensée en plusieurs sous cadres, qui entraîne la coexistence de conceptions concurrentes.

Comme l'écrivent Johsua et Dupin (1993), cette parcellisation en «registres ${ }^{1}$ de fonctionnement cognitif » peut aboutir à :

«de très fortes contradictions logiques entre les raisonnements utilisés dans chaque cas, un raisonnement pouvant, par exemple, et en toute rigueur, invalider l'autre. Mais alors que l'exigence de cohérence interne est constitutive du discours scientifique, elle ne l'est pas au même titre dans le comportement d'un individu donné. La règle ici est au contraire celle de la cohabitation de conceptions différentes, relativement étanches entre elles, correspondant à une classe de problèmes semblables (au yeux de l'élève), conceptions qui appellent des registres différents de fonctionnement cognitif».

La figure qui suit (Fig. 3) schématise cette modélisation en faisant apparaître un cadre culturel de rationalité comme une instanciation disciplinaire du champ conceptuel (G. Vergnaud, 1992), et le cadre personnel du sujet comme la juxtaposition de sous cadres induits par les cadres culturels correspondants. Le point d'interrogation sur la flèche du bas pose la problématique de l'unification non uniforme de l'espace de pensée du sujet.

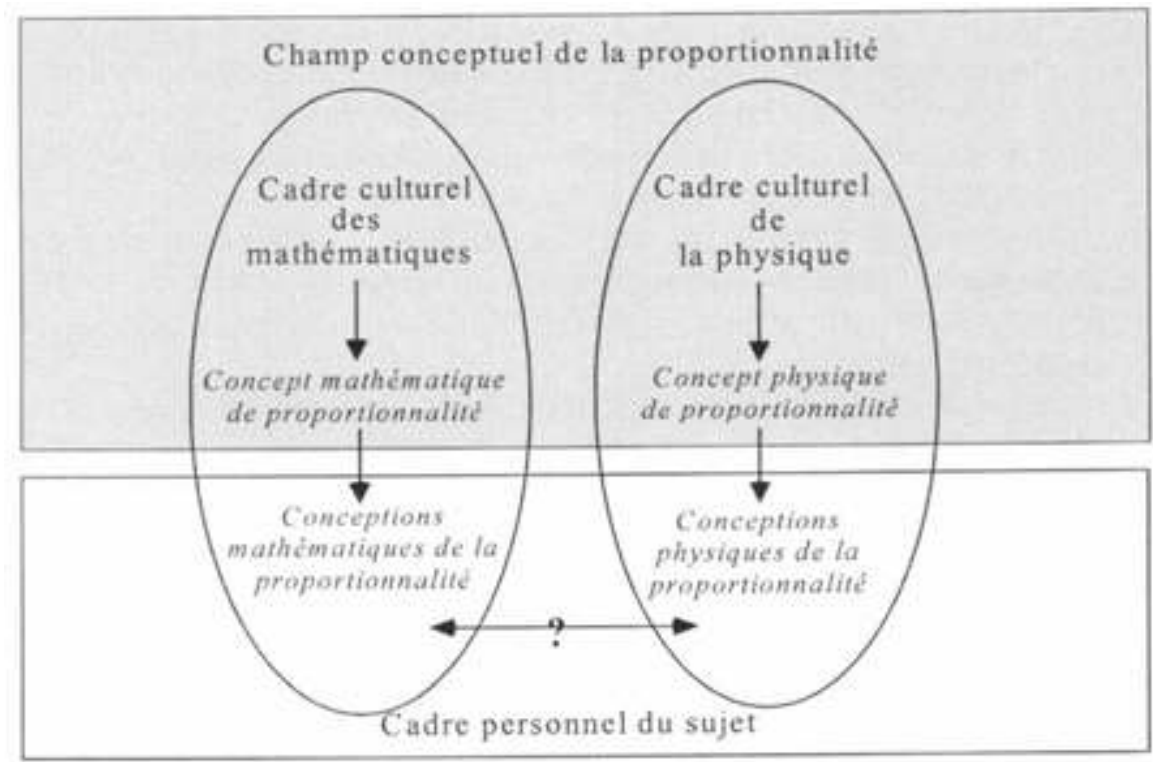

Figure 3

Champ conceptuel et cadres de rationalité 
Ce schéma fait apparaître une différenciation de vocabulaire entre concept et conception. Au sens de M. Bunge (1983), nous avons distingué les « objets conceptuels » produits par la culture (ici les concepts) des " objets mentaux " pensés par le sujet (ici les conceptions). Nous avons généralisé cette dualité à l'ensemble du vocabulaire utilisé (A. Lerouge, 2000). En corollaire, dans la ligne de la classification de M. Bunge, nous avons défini les «objets matériels » comme des objets hors du cadre psychique et du cadre culturel, ce qui nous a amenés à introduire la notion « d'espace de réalité ».

\section{L'espace de réalité}

Prenons un exemple simple, d'objet dit "matériel » : un tuyau de cuivre. Cet objet de la réalité n'est pas perçu de la même façon par un plombier qui peut le considérer comme un morceau de canalisation, par un électricien qui peut l'imaginer comme un élément de circuit électrique, ou encore par un hooligan qui envisage de s'en servir comme d'une arme. Cet exemple montre bien la différence entre l'objet matériel et l'objet mental, et ouvre la problématique de la réalité. Notre position épistémologique a été de postuler l'existence d'un « espace de réalité » défini comme l'ensemble des objets réels et des événements existant hors de la pensée personnelle ou collective, et sur lequel portent à la fois l'activité psychique des individus et l'activité de réflexion des communautés culturelles. Dans cette perspective, la conceptualisation apparaît comme une «projection» de l'espace de réalité dans un cadre personnel de rationalité via le filtre de divers cadres culturels (D. Malafosse, 1999).

\section{Exemples de mise en œuvre coordonnée des notions de registre sémiotique et de cadre de rationalité dans une perspective inter-didactique}

21 Le schéma qui suit (Fig. 4) présente de manière synthétique l'articulation des notions de registre sémiotique et de cadre de rationalité que nous avons mis en œuvre dans une perspective inter-didactique. Spécifié à l'apprentissage de la loi d'Ohm, il fait apparaître le registre graphique et le registre algébrique comme registres fondamentaux de cet apprentissage, et les démarches antinomiques induction/ déduction qui marquent la rationalité des cadres mathématique et physique. Sur la base de cette modélisation, nous avons travaillé les continuités et les ruptures de registres sémiotiques entre ces deux cadres de rationalité. Nous donnerons pour exemple deux extraits de nos expérimentations: un débat entre des PLC2 de mathématiques et de physique et des activités d'élèves lors d'une séquence en classe de Troisième. 


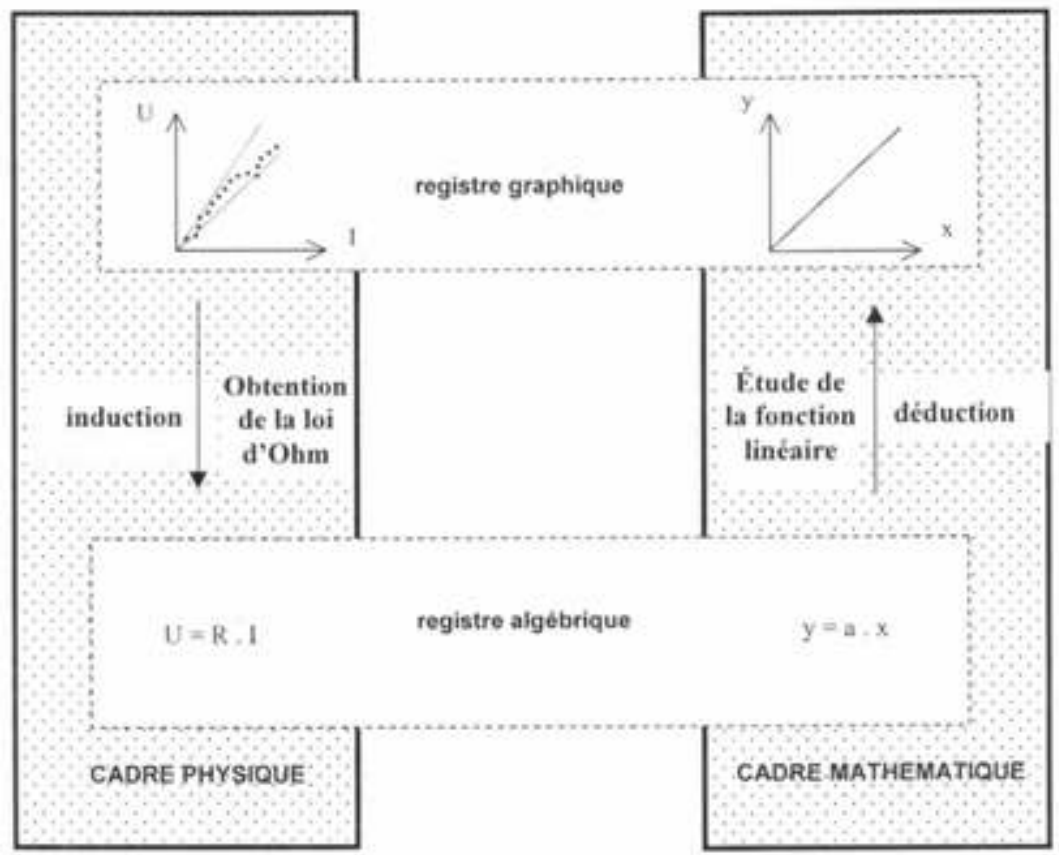

Figure 4

Coordination des notions de cadre de rationalité et de registre sémiotique.

\section{Analyse d'un débat entre des PLC 2 de mathématiques et de physique}

Dans une première expérimentation, nous avons organisé et filmé deux débats regroupant chacun vingt professeurs stagiaires de sciences physiques et de mathématiques à partir d'un questionnaire relatif à la représentation cartésienne utilisée en fin de Troisième, tant dans le cadre des mathématiques (représentations de fonctions) que dans celui des sciences physiques (caractéristiques de dipôles, etc.). Ce questionnaire était structuré en trois parties :

- définitions des objets géométriques qui apparaissent sur le graphique ;

- procédures d'obtention et traitement de ces objets ;

- statut de preuve d'un constat graphique.

L'analyse des débats nous a permis de faire apparaître les conceptions des membres des deux communautés, à propos des objets premiers de la géométrie et de leur utilisation en classe.

Les stagiaires de mathématiques sont capables de fournir "plusieurs définitions précises" de la droite ("par une équation cartésienne, par un point et une direction, par deux points, par une relation vectorielle ») et du point («par un ensemble de $\mathbf{n}$ coordonnées dans un espace à $\mathbf{n}$ dimensions, par l'intersection de deux droites»). Ces définitions n'ont pas, comme on pourrait s'y attendre, un caractère axiomatique, mais reposent, pour la plupart, sur le changement de registre algébrique/graphique.

Par contre, pour les physiciens, « la droite n'a pas besoin d'être définie » car " elle ne sert qu'à exploiter des points expérimentaux pour montrer une relation de linéarité » ou parce qu'elle n'est qu'un "cas idéal » et donc "n'existant pas », «la droite réelle n'étant en fait qu'un segment [...] dont les limites sont imposées par 
les contraintes matérielles", et "d'une épaisseur imposée par la précision des mesures ». Dans le même ordre d'idée, le point n'existe pas, car seule une "tache représente un état du système affecté de l'incertitude des mesures ». Cette position a été résumée par un des stagiaires de sciences physiques en réponse à une remarque de mathématicien qui craignait que le concept de droite ne soit confondu avec le trait du graphique par la phrase :

"Je regrette que mes élèves ne fassent pas des taches assez grosses et des bandes assez larges parce que, expérimentalement [...] je préférerais qu'ils fassent une tache qui couvre le domaine d'incertitude; là, il ferait de la physique. A partir de là, on passe à la notion de droite virtuelle et de point virtuel et on change de niveau ; on passe aux maths ».

Ainsi, il apparaît que les stagiaires des deux disciplines ont du mal à se comprendre lorsqu'on aborde le problème du statut de la droite qui « existe sans être représentée " pour les mathématiciens, alors qu'«elle n'est qu'un outil de travail» sans existence propre pour les physiciens. Ces écarts de conception nous ont amenés à comparer le registre graphique du cadre de rationalité de la physique à celui du cadre de rationalité des mathématiques, en nous centrant sur deux éléments figuratifs particuliers : le point et la droite. Le tableau ci-dessous donne une vision synthétique de nos analyses.

\begin{tabular}{|c|c|}
\hline \multicolumn{2}{|c|}{ Comparaison des registres graphiques en mathématiques et en physique } \\
\hline En physique & En mathématiques \\
\hline $\begin{array}{l}\text { Tout point graphique du plan représente un état potentiel- } \\
\text { lement accessible au système mesuré. A tout couple de } \\
\text { mesures (U, I) on associe une surface (tache) dont la } \\
\text { forme et laaire dépendent de la qualité de la mesure. }\end{array}$ & $\begin{array}{l}\text { A tout couple de nombres }(x, y) \text { on associe par bijection } \\
\text { un point graphique du plan XOY de coordonnées } x \text { et } y \text {. } \\
\text { Ce point a les propriétés géométriques d'un point } \\
\text { géométrique. }\end{array}$ \\
\hline $\begin{array}{l}\text { Les axes graphiques ont les propriétés géométriques des } \\
\text { droites orientées mais ils sont dimensionnés (par exemple, } \\
\text { l'axe des abscisses a pour dimension une intensité, alors } \\
\text { que l'axe des ordonnees est un axe de tension). }\end{array}$ & $\begin{array}{l}\text { Les axes graphiques ont aussi les propriétés géomé- } \\
\text { triques des droites orientées mais ils ne sont pas } \\
\text { dimensionnés. }\end{array}$ \\
\hline $\begin{array}{l}\text { La forme et l'aire de chaque tache dépendent des conven- } \\
\text { tions de mesure et de représentation (vecteurs unitaires } \\
\text { dont la longueur dépend du choix des unités de mesure, } \\
\text { convention de branchement des appareils de mesure). }\end{array}$ & $\begin{array}{l}\text { Le point reste un point par changement d'unité des axes } \\
\text { de coordonnées. }\end{array}$ \\
\hline $\begin{array}{l}\text { L'ensemble des taches graphiques représente l'ensemble } \\
\text { des états probablement accessibles au système, à la pré- } \\
\text { cision des mesures près. Les incertitudes expérimentales } \\
\text { ne permettent pas d'établir une relation de bijection entre } \\
\text { l'ensemble des points graphiques couverts par les taches } \\
\text { et l'ensemble des couples de mesures. La juxtaposition } \\
\text { des taches donne une autre tache encore affectée dincer- } \\
\text { titude. La propriété de continuité est occultée par celle } \\
\text { dimprécision des mesures. }\end{array}$ & $\begin{array}{l}\text { Lensemble des points graphiques est une droite gra- } \\
\text { phique. La tache graphique n'a pas de sens. }\end{array}$ \\
\hline $\begin{array}{l}\text { Pour des raisons de limites expérimentales et d'impréci- } \\
\text { sion des mesures, la bande est de longueur et d'epais- } \\
\text { seur finies. }\end{array}$ & $\begin{array}{l}\text { Les propriétés géométriques de la droite graphique sont } \\
\text { celles de la droite géométrique (longueur infinie, épaisseur } \\
\text { nulle, etc.). }\end{array}$ \\
\hline $\begin{array}{l}\text { Le passage de la tache au point graphique et de la bande } \\
\text { à la droite graphique se fait dans la perspective d'une idéa- } \\
\text { lisation des procédures expérimentales et par interpolation } \\
\text { lextrapolation de nature inductive des résultats expérimen- } \\
\text { taux idéalisés. Cette phase dídéalisation conserve aux } \\
\text { unités significatives du registre graphique des eléments } \\
\text { de rationalité du cadre de la physique. En particulier, tout } \\
\text { point graphique est affecté edautant de dimensions phy- } \\
\text { siques (tension et intensité) que de dimensions géomé- } \\
\text { triques (deux pour une représentation plane). }\end{array}$ & $\begin{array}{l}\text { Pour Duval (1988a), "la figuration d'un point relève d'une } \\
\text { autre procédure de représentation que celle de la droite: } \\
\text { elle est marquage d'une pure localisation, résultant d'une } \\
\text { pure visée deictique, ou de croisement de deux droites tra- } \\
\text { cées ". "Le tracé de la droite se génère dans un mouve- } \\
\text { ment et la propriété de continuité relève de cette } \\
\text { représentation dynamique". }\end{array}$ \\
\hline $\begin{array}{l}\text { Tant qu'elle n'est pas tracée, la droite graphique n'est pas } \\
\text { convertible dans le registre algébrique sous la forme d'une } \\
\text { relation fonctionnelle. C'est par construction de la droite } \\
\text { qu'on crée le signifié. }\end{array}$ & $\begin{array}{l}\text { La représentation droite graphique et sa conversion dans } \\
\text { le registre graphique sous forme d'une relation fonction- } \\
\text { nelle coexistent toujours. Le signifié correspondant existe } \\
\text { par définition. }\end{array}$ \\
\hline
\end{tabular}

Il apparaît sur ce tableau que les différences entre le registre graphique des mathématiques et celui de la physique se situent à la fois au niveau de la nature des signes et au niveau des règles de combinaison de ces signes pour produire des représentations. Pourtant, c'est sur l'illusion de continuité de ce registre que repose le jeu entre les mathématiques et la physique dans l'apprentissage de la loi d'Ohm lorsqu'on cherche à faire déterminer graphiquement la résistance d'un dipôle par lecture graphique du coefficient directeur de la « droite » $\mathrm{U}=\mathrm{f}(\mathrm{I})$. 


\section{Analyse de productions d'élèves} d'autres types de ruptures liées aux passages du cadre culturel des mathématiques à celui de la physique. En particulier, nous nous sommes intéressés à l'influence de la dimensionnalité des quantités physiques sur les règles de traitement du registre algébrique.

Nous avons proposé aux élèves le montage ci-contre (Fig. 5) composé d'un générateur de tension continue mais réglable, de deux dipôles ( 1 et 2 ), de divers appareils de mesure et des fils de connexion nécessaires.

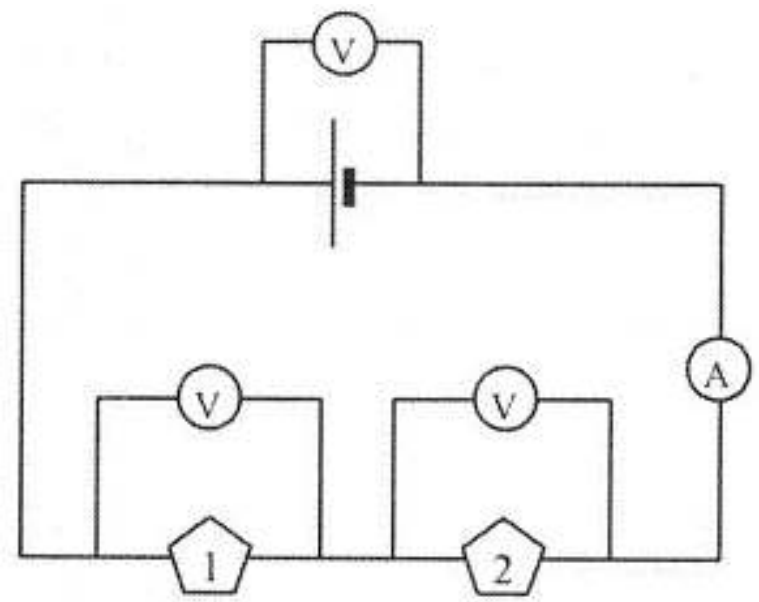

Figure 5

Montage circuit série présenté aux élèves.

Pour diverses valeurs de la tension du générateur, nous avons relevé, en classe, les indications données par chaque appareil de mesure et consignées dans le tableau cidessous.

\begin{tabular}{c|c|c|c|c}
\hline \multicolumn{6}{|c|}{ Tableau de mesures } & & & \\
\hline Groupes de mesures $\mathrm{N}^{\mathbf{2}}$ & $\mathrm{U}_{\text {générateur }}(\mathrm{V})$ & $\mathrm{U}_{1}(\mathrm{~V})$ & $\mathbf{U}_{2}(\mathrm{~V})$ & $\mathrm{I}(\mathrm{A})$ \\
\hline 1 & 0,0 & 0 & 0,00 & 0,00 \\
\hline 2 & 5,1 & 5 & 0,05 & 0,05 \\
\hline 3 & 10,8 & 10 & 0,70 & 0,10 \\
\hline 4 & 16,7 & 15 & 1,55 & 0,15 \\
\hline 5 & 23,1 & 20 & 2,90 & 0,20 \\
\hline 6 & 29,5 & 25 & 4,25 & 0,25 \\
\hline
\end{tabular}

L'examen attentif de ces données d'un point de vue purement numérique révèle une propriété surprenante, à savoir que la valeur de la tension du générateur est égale à la somme des tensions $\mathrm{U}_{1}$ et $\mathrm{U}_{2}$, augmentée de la mesure en ampères de l'intensité circulant dans le circuit. Ceci n'est pas le fruit du hasard mais est dû au fait que la résistance interne de l'ampèremètre a été délibérément choisie égale à $1 \mathrm{ohm}$.

Cette particularité va amener les élèves à travailler sur les deux relations : $U_{\text {générateur }=} \mathrm{U}$ ${ }_{1}+\mathrm{U}_{2}+\mathrm{I}$ et $\mathrm{U}_{\text {générateur } \approx} \mathrm{U}_{1}+\mathrm{U}_{2}$

31 La première relation, du fait de l'égalité stricte, est cohérente avec les règles de rationalité du cadre des mathématiques, mais n'est pas acceptable dans le cadre de 
rationalité des sciences physiques car elle ne satisfait pas aux règles d'homogénéité dimensionnelle. Par contre, la seconde, du fait de l'approximation des mesures met en défaut la rationalité des mathématiques. Nous avons demandé aux élèves de se prononcer sur la validité de ces deux relations.

Il s'avère qu'une écrasante majorité affirme sa préférence pour l'égalité stricte. Parmi les justifications proposées, on trouve de façon massive une remarque sur la hiérarchie entre les égalités strictes ou approximatives :

« La première loi est la meilleure parce qu'on a un signe = alors que l'autre n'est qu'une loi approximative $»$.

Ou encore sur la nécessité de l'exactitude de l'égalité :

"Une formule pas précise n'est pas juste».

D'autres facteurs interviennent aussi dans ce choix, notamment la nécessité de faire intervenir dans une formule toutes les données de l'énoncé, ou la nécessité de rapprocher toutes les quantités qui varient simultanément. Cependant l'analyse des justifications écrites et orales montrent que les raisons liées à la différence de nature dimensionnelle des quantités physiques sont totalement absentes des raisonnements qui conduisent à la discrimination de lois physiques à partir de relations numériques.

Les élèves traitent donc les données numériques expérimentales comme des nombres purs. Ils se placent ainsi dans le cadre des mathématiques pour établir des relations numériques. Ensuite, ils postulent implicitement la continuité de registre entre les deux cadres de rationalité pour transporter la relation dans le cadre de la physique.

\section{Conclusion}

Le modèle d'analyse des processus de conceptualisation que nous avons étudié permet d'aborder en termes de changements de cadres de rationalité et de changements de registres sémiotiques l'analyse inter-didactique de stratégies d'enseignement et d'apprentissage.

La mise en œuvre de la notion de registre nécessite de s'interroger sur la spécificité disciplinaire des registres sémiotiques. Nos exemples montrent en effet que la nature des éléments significatifs et des règles de traitement d'un registre dépendent tout autant de la nature du problème à traiter (et donc des règles de rationalité) que du registre lui-même. Il nous paraît difficile dans cette approche de séparer un registre du cadre de rationalité qui lui donne sens.

En particulier, dans l'étude du concept de résistance, via la loi d'Ohm, nous avons mis en évidence l'obstacle de non continuité du registre graphique cartésien et du registre algébrique ainsi que l'obstacle du changement de règles de raisonnement entre les deux cadres de rationalité. D'autres obstacles, liés à la difficulté que rencontrent les élèves pour coordonner des registres sémiotiques différents apparaissent à l'occasion de changements de registres dans un même cadre de rationalité. La conceptualisation scientifique ne s'arrêtant pas aux bornes des disciplines, il reste à concevoir des stratégies d'enseignement dépassant la dimension disciplinaire pour traiter sur le fond les problèmes que nous avons soulevés. 


\section{BIBLIOGRAPHIE}

DOUADY R. : Jeux de cadres et dialectique outil-objet. Thèse d'État, Université Paris VII, 1984.

DOUADY R. : « Des apports de la didactique des mathématiques à l'enseignement », in Repères IREM.Nº, pp. 132-158, 1992.

DUVAL R. : « Écarts sémantiques et cohérence mathématique : introduction aux problèmes de congruences ", in Annales de Didactique et de Sciences Cognitives de l'IREM de Strasbourg, $\mathbf{N}^{\circ} 1$, pp. 7-25. Strasbourg, IREM de Strasbourg, 1988.

DUVAL R. : «Quel cognitif retenir en didactique des mathématiques ? ", in Actes de la VIII École d'été de Didactique des Mathématiques.pp. 198-214, 1995a.

DUVAL R. : Sémiosis et pensée humaine. Berne (Suisse), Peter Lang, 1995b.

GIORDAN A. \& DEVECCHI G. : Les origines du savoir. Des conceptions des apprenants aux concepts scientifiques. $2^{\mathrm{e}}$ éd., 1990. Neuchâtel (Suisse) et Paris, Delachaux \& Niestlé, 1987.

JOHSUA S. \& DUPIN J.-J. : Introduction à la didactique des sciences et des mathématiques. Paris, PUF, 1993, pp. 127-128.

LEROUGE A. : Représentation cartésienne, rationalité mathématique et rationalité, du quotidien chez des élèves de collège. Thèse de doctorat, Université Montpellier II, 1992.

LEROUGE A. : « La notion de cadre de rationalité. A propos de la droite au collège. », in Revue Recherches en Didactique des mathématiques, $\mathrm{Vol}$ 20, $\mathrm{N}^{\circ} 2$, pp. 171-208, 2000.

MALAFOSSE D. : Contribution à l'analyse et à la modélisation des processus de conceptualisation en interdidactique des mathématiques et de la physique : exemple de la loi d'Ohm. Thèse de didactique des disciplines scientifiques, Université Montpellier II, 1999.

MALAFOSSE D., LEROUGE A. : « Ruptures et continuités entre physique et mathématique à propos de la caractéristique des dipôles électriques linéaires ", in Aster, № 30, pp. 65-85, 2000.

MALAFOSSE D., LEROUGE A. \& DUSSEAU J.-M. : « Etude en inter-didactique des mathématiques et de la physique de l'acquisition de la loi d'Ohm au collège : Espace de réalité. », in Didaskalia, $\mathbf{N}^{\circ}$ 16, pp. 81-106, 2000.

MALAFOSSE D., LEROUGE A. \& DUSSEAU J.-M. : « Etude en inter-didactique des mathématiques et de la physique de l'acquisition de la loi d'Ohm au collège. Changement de cadres de rationalité. ", in Didaskalia, $\mathbf{N}^{\circ} 18,2001$ a. (à paraître).

VERGNAUD G. : « La théorie des champs conceptuels », in Recherches en Didactique des Mathématiques, Vol 10, N² 2-3, pp 133-170, 1990.

VERGNAUD G. : « Homomorphisme réel - représentation et signifié - signifiant », in Didaskalia, $\mathbf{N}^{\circ}$ 5, pp. 25-34, 1994.

VERGNAUD G. : « Au fond de l'apprentissage, la conceptualisation. », in Actes de la VII École d'été de didactique des mathématiques,pp. 174-185, 1995.

VIENNOT L. : Raisonner en physique : la part du sens commun. Bruxelles (Belgique), DeBœk, 1996. 


\section{NOTES}

1. Le terme " registre » employé par ces auteurs recouvre dans notre terminologie la notion de cadre personnel de rationalité.

\section{RÉSUMÉS}

La question de déterminer sous quelles conditions des concepts élaborés dans le champ de la didactique d'une discipline donnée sont transposables à la didactique d'une autre discipline est régulièrement posée par de nombreux chercheurs. Pour notre part, nous avons tenté de répondre à cette question dans le cas particulier du transfert, en didactique de la physique, de quelques concepts mis en oeuvre en didactique des mathématiques pour analyser les processus de conceptualisation. Cette tentative nous a conduits à élaborer un modèle que nous qualifions d'inter - didactique car permettant de modéliser les processus de conceptualisation dans les deux disciplines. Il s'appuie sur les notions de cadre et de registre sémiotique développées de manière spécifique en didactique des mathématiques, et sur une notion nouvelle, celle d'espace de réalité, dont la nécessité s'impose de façon évidente en didactique de la physique, mais dont nous montrons l'utilité en didactique des mathématiques.

The issue is repeatedly raised by numerous researchers of determining whether, how and under what conditions, it is possible for concepts developed within the field of the didactics of a given discipline to be transposed to the field of the didactics of another. For our part, we have endeavoured to address this issue in the specific case of the transfer to the didactics of physics of a few concepts developed within the field of the didactics of mathematics, in order to analyse the conceptualisation processes involved. This endeavour led us to develop a model which we terra "inter-didactic" for it allows one to model the conceptualisation processes of both disciplines, This model is based on the notions of semiotic framework and semiotic "register", specifically as these concepts are developed in the didactics of mathematics, as well as on a new notion, that of "spatial view of reality". The need for this new concept imposes itself so to speak, obviously, in the field of physics, but we demonstrate its usefulness in the didactics of mathematics also.

\section{INDEX}

Keywords : inter - didactic, mathematics, physics, rationality framework, register, spatial view of reality, teacher training

Mots-clés : cadre de rationalité, espace de réalité, formation des maîtres, inter - didactique, mathématiques, physique, registre 


\section{AUTEURS}

\section{DIDIER MALAFOSSE}

Groupe GRIMP (Groupe de Recherches en Inter-didactique des Mathématiques et de la Physique), laboratoire ERES (Etudes et Recherches sur l'Enseignement Scientifique - EA 730), Université

Montpellier II

\section{ALAIN LEROUGE}

Groupe GRIMP (Groupe de Recherches en Inter-didactique des Mathématiques et de la Physique), laboratoire ERES (Etudes et Recherches sur l'Enseignement Scientifique - EA 730), Université Montpellier II

JEAN-MICHEL DUSSEAU

Groupe GRIMP (Groupe de Recherches en Inter-didactique des Mathématiques et de la Physique), laboratoire ERES (Etudes et Recherches sur l'Enseignement Scientifique - EA 730), Université Montpellier II 